\title{
AN ARTIFICIALLY GENERATED ELECTRODE EFFECT AT GROUND LEVEL
}

\author{
S.Israelsson and E.Knudsen \\ Department of Meteorology, Uppsala University \\ Uppsala, Sweden
}

\begin{abstract}
Outdoor experiments show that an artificially produced electrode effect in the atmospheric surface layer can easily be built up and broken down in less than 7 seconds. The wind speed, the external electric field and the ion mobility are the most dominant factors in building up the electrode layer.
\end{abstract}

\section{Introduction.}

The electrical processes in the planetary boundary layer are complex, and vary within a large range of space and time scales. An overview of electric phenomena in the lower atmosphere is given by Hoppel et al. (1986).

A source of space charge at ground level is the electrode effect (e.g. Israel, 1971). The mathematical description of the electrode effect under convection and turbulent mixing is given by a number of authors (Chalmers, 1967; Hoppel, 1967; Willett, 1978, 1979, 1983; and Tuomi, 1982). However, the results of numerous experiments are confusing. Chalmers (1967) reported that "the simple electrode effect does not occur". Mühleisen (1961) reported a well-pronounced effect over water, but none over land. Bent and Hutchinson (1966) concluded that "the electrode effect made itself felt in a number of different ways". Crozier (1963) found "a strong manifestation of the electrode effect" during nighttime periods with a very low wind velocity.

This disparity can be accounted for, in part, by the different measuring methods and the varying local meteorological conditions during the measurement. Varying surface radioactivity (trapped radon and thoron) and aerosol concentration give differing results as well. Another complication is the large signal fluctuations during the measurement periods, observed by several researchers (Israel, 1973).

A survey of the present knowledge of the electrode effect, i.e. the imbalance between small positive and negative atmospheric ions caused by the atmospheric electric field in the surface layer together with strong evidence for the existence of this effect over snow-covered ground has been given by Knudsen et al.(1989).The magnitude of the effect depends upon several parameters; namely the atmospheric electric field, the mobility of the ions, the ionization rate, the recombination of the ions and the attachment effect of ions to the ground. Besides, meteorological factors such as turbulence and stability of the atmosphere will play a dominant role. Most of the above mentioned factors can vary within a wide range, which makes comparisons between theoretical models and experimental results difficult.

The space charge density consists of two components (see e.g. Knudsen and Israelsson,1994); - small-ions, transported by air movements and by the influence of the atmospheric electric field, and large ions transported by air movements only.

It is the intention of this paper to present an experimental investigation of the electrode effect in the atmospheric surface layer under controlled electric field and wind conditions. 


\section{Measurement site and experimental setup}

The measurements were made at the Marsta Observatory $\left(59^{\circ} 55^{\prime} \mathrm{N}, 17^{\circ} 35^{\prime} \mathrm{E}\right)$. The observatory is located in very flat farming country area $10 \mathrm{~km}$ north of Uppsala, Sweden. The nearest forest is more than $1 \mathrm{~km}$ away from the observatory, which yields undisturbed micrometeorological conditions. No industrial establishments produce condensation nuclei in the surroundings of the observation place and no burning took place during the observations. A map of the Marsta Observatory is presented by Israelsson et al. (1973).

The micro-meteorological parameters, wind, temperature and relative humidity were continuously recorded with standard instruments.

The atmospheric electric potential gradient was measured with a radioactive collector method at a height of $1 \mathrm{~m}$ and the electric polar conductivity $\left(\lambda^{+}\right.$and $\left.\lambda^{-}\right)$at a height of $0.5 \mathrm{~m}$ with two aspiration condensers according to the Kasemir-Dolezalek system (Israel, 1971 and 1973).

The aerosol particles were measured with a Gardner condensation nuclei counter $0.5 \mathrm{~m}$ above the ground surface. In the investigation a metal grid $\left(10 \times 4 \mathrm{~m}^{2}\right)$ with mesh dimension 8 $x 8 \mathrm{~cm}^{2}$ was used, - placed on insulating poles of $1.5 \mathrm{~m}$ height, as shown in Figure 1.

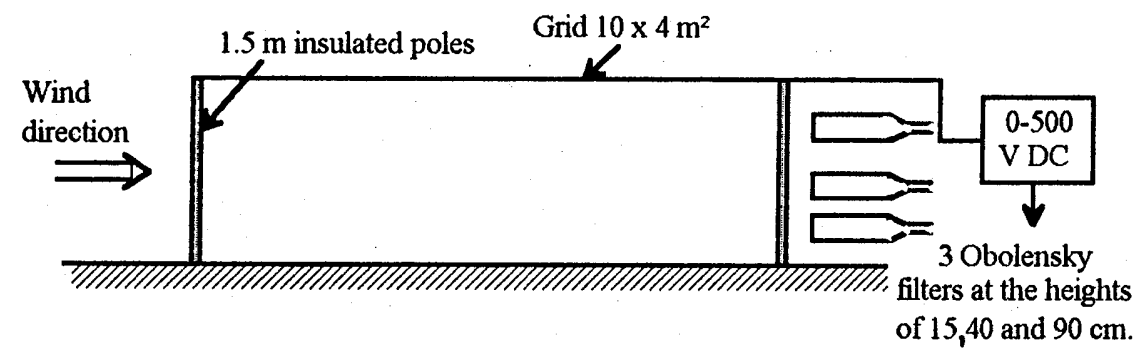

Fig.1. Experimental setup.

The grid was situated on an open flat field well apart from obstacles disturbing the wind. The ground was covered with short cut grass, ca. $5 \mathrm{~cm}$ both under the grid and ca. $5 \mathrm{~m}$ in front of the grid. A voltage could be applied to control the field between the grid and ground; in the present investigation the values 0,100 and $200 \mathrm{Vm}^{-1}$ were used. With three identical Obolensky filters, at the heights of $0.15,0.40$ and $0.90 \mathrm{~m}$ at the lee side of the grid, the space charge density profile was measured. With some minor modifications the filters are a copy of the construction given by Anderson (1966). The instruments have been used in other investigations with good results. To ventilate the filters three standard vacuum cleaners were used. The output currents from the Obolensky filters were measured in electrometer amplifiers operating as current to voltage converters. The time constant was adjusted to 3 seconds. By using the Obolensky filters the total space charge density measured, both small and large ions.

Simutaneous recordings of the three instruments were logged during three-minute periods, a total of 400 values, and means were calculated. Due to the small differences between the individual gradient values of the space charge density, very high sensitivity was required.

Even if the instruments were equally constructed the sensitivity could not be expected to be the same. This was controlled in the following way. The three instruments were placed next to each other at the same height and 400 values were recorded for 3 minutes. The sensitivities of the instruments were closely checked to be equal. Offset controls and the calibration 
adjustment routines were run frequently during the recording periods.

It should be desirable to measure the space charge profile in front of the grid. The measurement at the lee side of the grid was however very time consuming. Considering the very small differences in space charge densities $\left(100-400\right.$ elementary charges per $\left.\mathrm{cm}^{3}\right)$ for the different levels, the stability of the instruments had to be frequently checked with respect to thermal drift and sensitivity. Besides several profiles have to be recorded during same meteorological conditions, especially same wind direction.

In a previous investigation (not published results) the space charge density profiles have been investigated for different stability and wind conditions. The method of measuring was described by Knudsen et al. (1989). One of the measured profiles fitted well with the micrometeorological conditions of the present investigation and the profile shows a well developed electrode effect. It is therefore motivated to assume that in the present study an electrode effect was present in front of the grid. For comparison this profile has been included in the figures, see Figures 2 and 3.

3. Recordings and experimental results.

As the recordings were very time-consuming, reliable measurement required:

- Stable meteorological conditions for several hours.

- Steady wind direction.

- Frequent calibration and offset adjustments.

- Preferably several runs to calculate the mean values.

All recordings were carried out during 2 days under fair weather conditions with no clouds, dry ground surface, visibility $>50 \mathrm{~km}$, low to moderate wind speed and temperatures $12-15^{\circ}$ C. On both days the atmospheric electric field was about $80 \mathrm{Vm}^{-1}$ with a fluctuation of \pm 10 $\mathrm{Vm}^{-1}$. The aerosol particle concentration was about $2000 \mathrm{~cm}^{-3}$.

After recordings of four double profiles (1600 values on each level) on October 7, 1991, the wind speed decreased and two additional profiles were recorded under the otherwise same conditions. The space charge density profiles are shown in Figure 2.

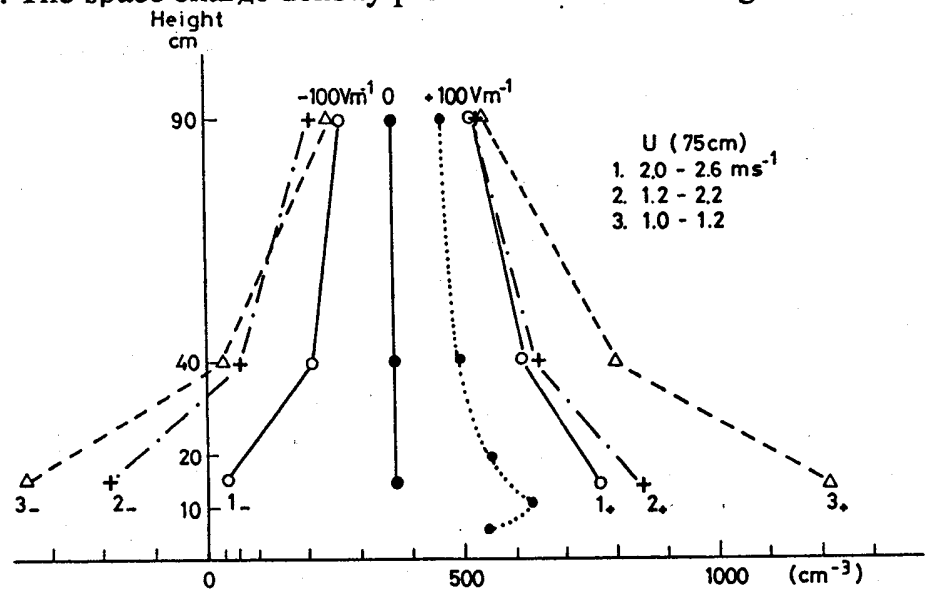

Fig. 2. Space charge density profiles during different wind speeds and electric fields. The dotted curve represents measurements without the grid. 
On September 26, 1991 (Figure 3), the wind speed was the same as for profile 1 shown in Figure 2, but a twice as high field was applied to the grid. Altogether 6 double profiles were recorded, and the mean profiles are shown in Figure 3 .

The vertical profiles in Figures 2 and 3 show a well-defined decrease of the space charge density with increasing height for different applied electric field.

The space charge density for zero voltage at the grid differs only slightly from one profile to another. The differences were so small that for better clarity they were adjusted to the same value in the diagrams.

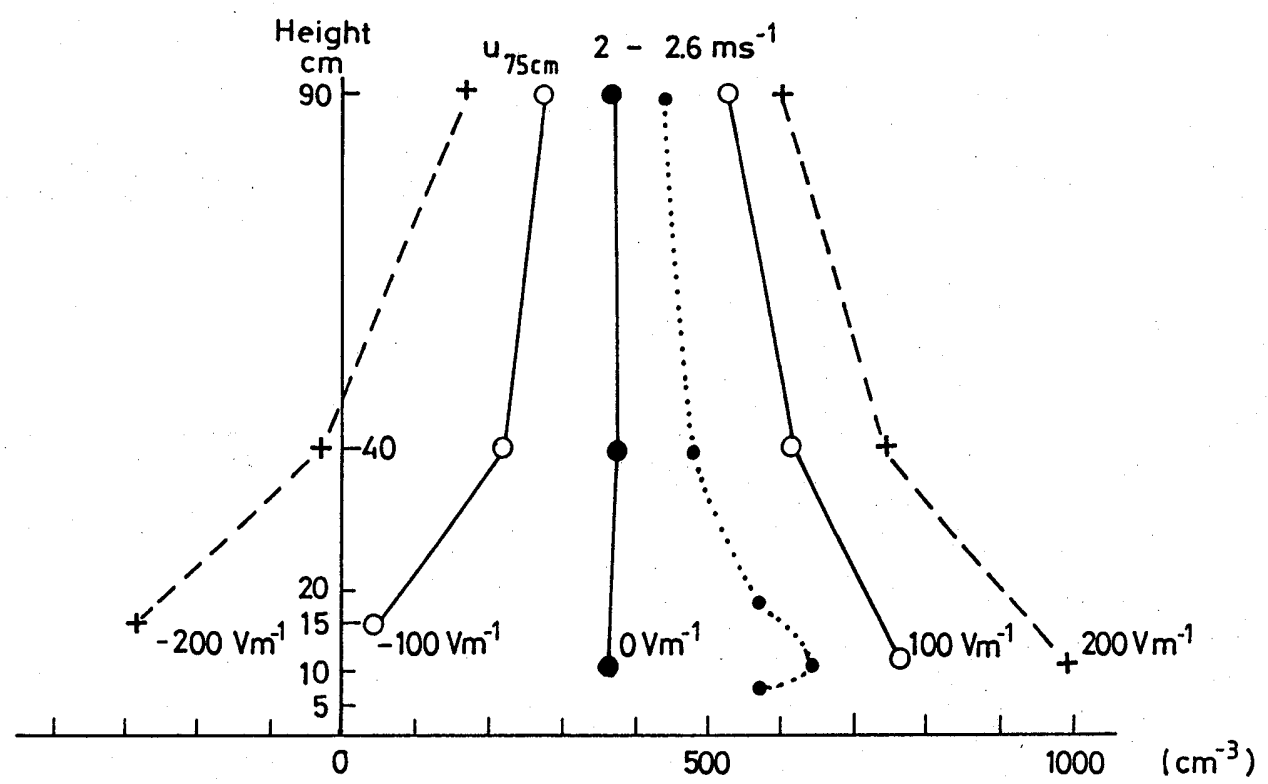

Fig.3. Mean space charge density profiles. The dotted curve represents measurements without the grid.

We consider that for zero voltage at the grid no ion separation takes place in the air passing under the grid. The combination and recombination of ions is very limited during the passage and the profile recorded is equivalent to that entering the grid. The diagrams show that the vertical gradient is close to zero. This means that the presumed electrode effect at the inlet of the grid (cf. Figure 1) is eliminated under the metallic grid by the turbulence. Small deviations are within the accuracy of measurements.

An electrode effect could have existed in front of the grid, but it is obvious that the gradients shown in the diagrams are generated during the time of air-passage under the grid. The times required to generate the space charge profiles in Figure 2 are roughly 7,10 , and 15 seconds. The values are calculated from assuming a logarithmic wind profile below the grid and then by using a mean wind speed. Seven seconds is apparently time enough to eliminate a presumed electrode effect at the front of the grid.

Recordings with an Obolensky filter include the total space charge density regardless of the mobility of the ions. Space charges consists of two components (see e.g. Knudsen and Israelsson, 1994), - small ions of high mobility and large ions of low mobility. The transport 
mechanisms of the two types differ basically. Small ions will be transported by the influence of the atmospheric electric field and by air movements, whereas large ions will mainly be transported by air movements. In all the diagrams the space charge density will include both components, but only the small ions will contribute to the space charge gradients. The large ions are assumed to be constant with height due to a low electric mobility and therefore not dependent on the electric field strength.

In Figures 2 and 3, the space charge profiles for zero field are in the positive range, which can be explained by the occurrence of a surplus of positive large ions in the lowest layer of the atmosphere due to the formation of positive small-ion space charges in the electrode effect layer. The large ions are mainly transported by air movements and not influenced by the electric field.

The ion separation in an electrode effect results in a vertical component of the gradient of the space charge density, i.e. $\mathrm{d} \rho / \mathrm{dz}$, or here $(\mathrm{dp} / \mathrm{dz})_{1}=\left(\rho_{0.4}-\rho_{0.15}\right) / 0.35$ and

$$
(\mathrm{d} \rho / \mathrm{dz})_{2}=\left(\rho_{0.9}-\rho_{0.4}\right) / 0.5 \text {. }
$$

By measuring $\mathrm{d} \rho / \mathrm{dz}$ for the two cases, constant field with different wind speeds (Figure 2) and the same wind speed with different fields (Figure 3), the influence of the two parameters can be determined. By increasing the field from 100 to $200 \mathrm{Vm}^{-1}$ we found the increase of the gradient to be a factor of about 2 . By increasing the wind speed by a factor of 2 the gradients decrease with a factor of about 2.5 . The ratios were about the same for both polarities.

\section{Discussion}

The experiments with a metallic grid above the ground surface yielded very well-defined vertical profiles of the space charge density. The profiles showed a strong evidence for the existence of an electrode effect in the atmospheric surface layer. The build up or break down of an electrode-effect layer occurs in less than 7 seconds.

The electrode effect is dependent on the electrical field strength, turbulence, and ion mobilities. Wind speed seems to be somewhat more dominant than the other factors in the building up of the electrode layer. This can be explained in the following way. When the field is increasing, all other parameters which contribute to the ion separation will be unchanged and only effects of the field strength will be observed. On the other hand, - increasing wind speed results in growing turbulent exchange together with shorter times for the ions to be exposed to the applied electric field.

A comparison between the space charge density gradients given in the present study and theoretical calculations of the electrode effect model given by Tuomi (1982) is made. The Tuomi model, which is mainly valid for cases with snow-covered ground, includes turbulent ion transport, and makes a linearization of the steady state equations that govern the atmospheric electricity parameters near plane ground (Willett, 1978). It is assumed that aerosols are uniformly distributed and only effect the order of magnitude of the small-ion densities. The effect of aerosol was taken into consideration by use of an effective value of the ionization rate, $q$, viz:

$$
\mathrm{q}_{\text {eff }}=\mathrm{q}-\beta \mathrm{nN}=\alpha \mathrm{n}^{2}
$$

where $\mathrm{n}=\mathrm{n}^{+} \approx \mathrm{n}^{-}$is the small-ion density. $\mathrm{N}$ is the aerosol density. We used $\mathrm{q} \approx 10^{7} \mathrm{~m}^{-3} \mathrm{~s}^{-1}$ 
the true ionization rate and $\alpha \approx \beta \approx 1.6 \cdot 10^{-12} \mathrm{~m}^{3} \mathrm{~s}^{-1}$ the small-ion recombination and aerosol attachment coefficients, respectively. The small-ion density, $n$, can be calculated from the conductivity, $\lambda$, by:

$$
\lambda=2 \mathrm{kne}
$$

where $\mathrm{k}$ is the mobility; in our calculation $1.4 \cdot 10^{-4} \mathrm{~m}^{2} \mathrm{~V}^{-1} \mathrm{~s}^{-1}$.

For the actual conditions we used the same electrical fields as in the experiment; roughness parameter $z_{0}=0.01 \mathrm{~m}$ and $\mathrm{u}^{*}=0.07$ and $0.15 \mathrm{~ms}^{-1}$ calculated from the logarithmic wind law.

Comparison between calculated and experimental gradients of the space charge density for different electric field strengths and wind speeds agree very well, see Table 1 . By increasing the field from 100 to $200 \mathrm{~V} / \mathrm{m}$ we found from the calculations an increase of the gradient by a factor of 2 , which is the same value as the measured one. By increasing the wind speed by a factor of 2 the gradients decrease with a factor of 2.6 , which is close to the experimental value 2.5.

The agreements between the experimantally and theoretically obtained gradient data show that the obtained profiles are results after completion of equilibrium.

Table 1. The space charge density difference between the levels 0.15 and $0.9 \mathrm{~m}(\Delta \rho)$ for different wind speeds and electric field strengths.

\begin{tabular}{ccccc}
\hline $\mathrm{U}^{*}\left(\mathrm{~ms}^{-1}\right)$ & $\mathrm{E}\left(\mathrm{Vm}^{-1}\right)$ & $\Delta \rho_{\text {theor. }}\left(\mathrm{cm}^{-3}\right)$ & $\mathrm{U}_{(75 \mathrm{~cm})}\left(\mathrm{ms}^{-1}\right)$ & $\Delta \rho_{\mathrm{exp} .}\left(\mathrm{cm}^{-3}\right)$ \\
\hline 0.07 & 100 & 440 & $1.0-1.2$ & 580 \\
0.15 & 100 & 170 & $2.0-2.6$ & 230 \\
0.15 & 200 & 330 & $2.0-2.6$ & 460 \\
\hline
\end{tabular}

The observed differences of the absolute values in Table 1 depend on the difficulty to use correct input data, (wind and ionization profiles, electric mobility etc), in the theorertical model.

The destruction of the natural electrode effect in front of the grid can be explained both by the turbulent mixing of the air passing under the grid and by the absence of ion separation due to no electric field.

The build up of a well developed electrode effect during less than 7 seconds cannot be explained by the theory of the classical electrode effect. Using the approximate equation of the relaxtion time $T$ of the atmosphere given by Israel (1971), which is valid in pure laminar motion, the actual value of $\mathrm{T}$ will in the present case be $730 \mathrm{sec}$. for a total conductivity of 50

$\mathrm{fSm}^{-1}$. This value is hundred times greater than the measured one. The observed time required for annihilation of the natural electrode effect and for setup its artificial counterpart are much shorter than either the electrical relaxation time or presumably the small-ion lifetime in the medium of about 2000 aerosol particles per $\mathrm{cm}^{3}$. Therefore, the dominant physical processes in the experiment are turbulent diffusion and drift in the applied field of ambient small ions that have been advectived beneath the screen from upstream.

By the present method it will be possible in future studies to shed much light to one important question, the non-existence or smallness of the natural electrode effect over grass surface. By using data on the vertical distribution of natural radioactivity under different 
stability conditions the influence of the ionization on the electrode effect can be studied more penetratingly. Further ion mobility, aerosol attachment and large-ion charge density play major roles in the natural electrode effect which can be included in future studies.

Acknowledgements. The study was supported by grants from the Swedish Natural Science Foundation

\section{References}

Anderson, R.,A., Absolute measurements of atmospheric charge density, J. Geophys. Res., 71, 5809-5814, 1966.

Bent, R.,B. and W.C.A. Hutchinson, Electric space charge measurements and the electrode effect within the height of a $21 \mathrm{~m}$ mast, J. Atm. Terr. Phys., 28, 53-73, 1966.

Chalmers, J.,A., Atmospheric Electricity, 2nd ed., Pergamon Press, Oxford, 1967.

Crozier, W.,D., Electrode effect during night-time low-win conditions, J. Geophys. Res., 68, 3451-8, 1963a.

Dhanorkar, S., and A.K. Kamra, Measurement of mobility spectrum and concentration of all atmospheric ions with a single apparatus, J. Geophys. Res., 96, 18671-18678, 1991.

Hoppel, W., Theory of the electrode effect, J. Atm. Terr. Phys., 29, 709-721, 1967.

Hoppel, W., R.V. Anderson, and J.C. Willett, Atmospheric electricity in the planetary boundary layer, in The Earth' Electrical Environment, Studies in Geophysics, National Academy Press, USA, 149-165, 1986.

Israel, H., Atmospheric Electricity I, U.S. Dept. of Commerce, Springfield, 1971 .

Israel, H., Atmospheric Electricity II, U.S. Dept. of Commerce, Springfield, 1973.

Isralesson, S., E. Knudsen, and E. Ungethum, On the natural $\beta$-activity of the air in the atmospheric surface layer, Atm. Environment, Pergamon Press, I, 1127-1137, 1973.

Knudsen, E., and S. Israelsson, Mobility spectrum of ions in the electrode layer, J. Geophys. Res., 29 , in press, 1994.

Knudsen, E., S. Israelsson, and B. Hallberg, Measurements of the electrode effect over flat, snow-covered ground, J. Atm. Terr. Physics, 51, 521-527, 1989.

Muhleisen, R., Electrode effect measurements above the sea, J. Atm. Terr. Phys., 20, 79-81, 1961.

Tuomi, T.,J., The atmospheric electrode effect over snow, J. Atm. Terr. Phys., $\underline{44}$ 737-746, 1982.

Willett, J.,C., An analysis of the electrode effect in the limit of strong turbulent mixing, J. Geophys. Res., $\underline{83}$, 402-408, 1978.

Willett, J.,C., Fair weather electric charge transfer by convection in an unstable planetary boundary layer, J. Geophys. Res., 84, 703-718, 1979.

Willett, J.,C., The turbulent electrode effect as influenced by interfacial ion transfer, J. Geophys. Res., 88, 8453-8469,1983. 